\title{
An Urgent Communication Way of Utilizing IOT Sensor for the Disabled
}

\author{
Donghyeok Suh ${ }^{1}$, Chang-Won Seo ${ }^{2}$ and Kwang-Chul Park ${ }^{3}$ \\ ${ }^{1} 119$, Dandae-ro, Dongnam-gu, Cheonan-si, Chungnam, 31116, Korea \\ 276-32, Daehakgil, Kamgok-myun, Eumsung-gun, Chungbuk, 27601, Korea \\ ${ }^{3} 48$, Wolbong-ro, Seobuk-gu, Cheonan-si, Chungnam, 31172, Korea \\ ${ }^{1}$ dhsuh122@dankook.ac.kr, ${ }^{2}$ cwseo@kdu.ac.kr, ${ }^{3}$ kcpark@kornu.ac.kr
}

\begin{abstract}
In this paper, we proposed a sensor application method that can support the urgent communication when the user is in an urgent situation. This study suggested how to use sensor data to quickly recognize and transmit to the outside of the handicapped who have difficulties in using generalized communication equipment or who are difficult to communicate quickly in an emergency. If a person with a physical disability is aware of the danger and avoids the risk, or urgently needs to express his intention by urgent necessity, even if this communication can be difficult, a solution should be prepared to solve this problem. Smart devices can be used for communication network connection and information processing together with sensors. The measured values of the three-axis acceleration sensor built in the generalized smart device such as smart phone or smart watch are obtained according to the time and classified by time. By making three measurement values of the three-axis acceleration sensor and comparing them by time zone, the disabled people inferred the urgent communication intention by recognizing the behavior pattern in the urgent situation and the intelligent propagation.
\end{abstract}

Keywords: disability, Communication, Internet of Thing, Sensor Data, Emergency situation

\section{Introduction}

Since 2016, a change called the 4th industrial revolution has proceeded in each industrial sector over the world. The existing leading industry is on the ebb, while new industry emerges as leading one, and an unprecedented change continues in each industrial sector. New patterns of production, distribution and consumption system are routinized, due to the 4 th industrial revolution. It is the recent information and communication fusion technology that enables such a change. The representative technologies include Internet of things(IoT), Big Data and Artificial Intelligence (AI). Although they are influential by themselves, more significant changes occur when they are fused with other fields. IoT, Big Data and AI can create smart medical fields, by fusion with medical technologies. The smart health care conceptually tackles conventional knowledge in the previous medical system and provides a new type of health care services.

The Internet of thing(IoT) can detect events in the real world without human intervention, process and analyze event data, and infer relevant real world situations. Various intelligent services can be realized based on the Internet of things. Sensor, data communication network, information processing device, and power supply unit are integrated so that they can be attached to various objects or widely distributed to enable intelligent situation recognition.

Received (October 15, 2017), Review Result (January 9, 2018), Accepted (January 31, 2018) 
Objects an important role in the IoT is the variety of sensors that detect physical, chemical, and biological changes as events and report them numerically. Utilizing a variety of different types of sensors helps to enhance the quality of information. IoT has a powerful function in reasoning and recognizing the real world situation, but the remarkable thing is the communication function. Compared with the previous ubiquitous system, data transmission is very stable. IoT has progressed in itself and has been used in various other fields to cause progressive change.

There is a marked difference between previous and smart health care. The former begins diagnosis and treatment only after a patient with a symptom visit a doctor, while the latter begins them, even though a symptom does not yet appear or a patient does not visit a doctor. This has not been admitted so far, according to common sense of medical system. Likewise, smart vehicles appeared, when IoT, Big Data and AI are converged with vehicles. They are not only breakthrough, in that they are conceptually different from the existing ones, but also innovative products which induce changes even in road and urban environment. Thus, IoT, Big Data and AI have resulted in very new and sensational changes across all industrial sectors.

Among them, IoT is the most central technology that leads the 4th industrial revolution. When IoT is applied, the technology and industrial fields are quickly turned into new modes. Such a change proceeds at unprecedented speed. Outcomes of the change fundamentally modified the existing technology and industry. When IoT is applied to a field, the field can secure intellectual performance. IoT enables a system itself to conduct intellectual judgment and management, with human interventions or administration. The introduction of IoT allows objects in real world to make adaptive decisions and do intellectual actions, which have been made possible only by human beings.

For IoT, the roles of networks are important. Network technologies used in IoT stably and intellectually transmit collected data at any adverse conditions. IoT can be also used as a tool to communicate or exchange information, in that it can intellectually continue to transmit data and maintain networks and it provides context-information by which contexts in real world can be remotely recognized. Therefore, IoT can be used as a communication tool in environment in which communication is not easily implemented or in the process of works characterized by difficult communication. Furthermore, it can be also used for people with communicative disorders.

In this study, we propose that can utilize IoT for emergency communication when an emergency situation occurs. The use of what can be used to recognize various complex situations in the real world as a simple communication tool is necessary for severely disabled people who are difficult to communicate and communicate. There are various types of disabilities. Some are physically healthy, others are difficult to communicate, and some have intellectual activity that does not interfere, but the physical function is significantly weakened. Some disorders are characterized by very partial disability of the body's function, but severe impairment or severe functional impairment is also present.

Recently, there have been fires in the homes of disabled people in Korea, but there have been cases where a person could not evacuate due to a physical disability that could have been evacuated sufficiently. The disabled person who had an accident was unable to evacuate even though he was able to act slowly, and did not have enough time to use the communication equipment. There are even more severe cases among people with physical disabilities, and in this case, communication needs to be secured in an emergency.

In this paper, we propose a sensor application method that can support the urgent communication when the user into a physically handicapped person is in an urgent situation. It is possible to utilize the sensor installed in the existing smart device rather than using a separate terminal combined with the sensor, the communication network and the information processing device. The sense of heterogeneity of the added portable terminal or the built-in type terminal may be felt to be larger than that of the non-disabled, so that the terminal may be detached. The advantage of using sensors that are built into 
existing smart devices is that they can be less heterogeneous and easier to recognize unusual events and abnormal activity situations based on routine activities.

This study suggests how to use sensor data to quickly recognize and transmit to the outside of the handicapped who have difficulties in using generalized communication equipment or who are difficult to communicate quickly in an emergency. To this end, a method for detecting the change of the user state based on the event information of the sensor is derived. It is to be able to recognize what is trying to communicate or convey urgent opinions without external help. A 3-axis acceleration sensor is built in to smart devices recently. Utilizing the data detected and reported by the three-axis acceleration is advantageous in reaching the research goal. The 3 -axis acceleration sensor is basically a sensor capable of measuring the gravitational acceleration in the $\mathrm{x}, \mathrm{y}$, and $\mathrm{z}$ axis directions. Using this sensor, you can find the slope of the object that the sensor is mounted on, and you can also express the angle and rotation about each axis. Collecting the measured values of the 3-axis acceleration sensor according to the time can realize a wide variety of applications. In this study, various information is deduced by analyzing pattern by classifying event information reported by 3 -axis acceleration sensor by time zone.

This study is composed as follows. In Chapter 2, related studies are summarized. In Chapter 3, we propose an emergency communication method for severely disabled persons who use the Internet of things. Chapter 4 describes the experiment and evaluation and concludes with the conclusions in Chapter 5.

\section{Related Research}

Lim, C.Y. et al., are turning into the aged society, accidents by falling down are increasing in the aged people's group. The system with the 3 -axis acceleration sensor is composed by a single chip. The body activity signal is measured with the signal detector and RF communicator in this proposed system and falling by the entering signal pattern analysis with 3 -Axis acceleration sensor. Human activities and falling is monitored according to analyze and judge the critical value of the Signal Vector. As fall down if they don't turn off the alarm after specific period and the aged person's after falling down activities are their position and more[1][9].

Jeon, K.M. et al., is to implement a multiple bio-signal central monitoring system based on textile fabrics flexible platform which can obtain and monitor bio signals(heart rate, body temperature, electrocardiography, electromyogram) of workers in special working environments and additional situational information (3axis acceleration, temperature, humidity, illumination, surrounding image). This system can prevent various accidents that may occur in the remote work environment and provide fast and efficient response by detecting workers' situations in real-time. For it, the textile fabrics flexible platform was made as innerwear or outerwear so that it does not interfere with workers' performance while collecting bio-signal and situational information, and obtained information is sent to the central monitoring system through wireless communication. The central monitoring system is based on wireless medical telemetry service of WMTS (Wireless Medical Telemetry Service) can monitor from 2 to 32 people simultaneously and was designed so that it can be expanded[2][10].

Kim, J.S. et al., implemented an indoor positioning system combining Android Smartphone, VLC(Visible Light Communication) using LED(Light Emitting Diode), and a cane for visually impaired people to provide them with indoor location information. System uses voice recognition function to input the destination and gathers location information from LED lighting through the visible light receiver module mounted on the cane. The cane and Smartphone exchange data using bluetooth and Smartphone guides the user to the destination by voice[3]. 
Kang, K.W. et al., developed HMI using electromyogram(EMG) and electrooculogram(EOG) for people with physical disabilities. Developed system is composed of two modules, hardware module for signal sensing and software module for feature extraction and pattern classification. To maximize ease of use, only two skin contact electrodes are attached on both ends of brow, and EOG and EMG are measured simultaneously through these two electrodes. From measured signal, nine kinds of command patterns are extracted and defined using signal processing and pattern classification method. System shows the capability of the developed system on real applications, five different types of commands are used to control ER1 robot. The results show that developed system can be applied to disabled person with quadriplegia as a novel interface way[4].

Kang, C.S. et al., propose a machine-to-machine based intelligent walking assistance system for safe and convenient walking of the visually impaired. system consists of a walking assistance stick used by the visually impaired and a server supporting multiple stick users in remote places through mobile communication networks. The stick equipped with ultra sonic sensors, GPS(global positioning system) receiver and vibrator not only detects floating obstacles, but also offers stick users with present location identification utilizing a text-to-voice conversion technology. Besides providing geographic information, the server notifies the emergency locations of users to guardian and aid agency, and it provides $\log$ information during walking such as the place, time and the number of accidents. Test results with a developed prototype system have shown that the system properly performs the functions and satisfies overall system performance[5].

Park, S.W. et al., reports web-based text-to-sign language translating system that helps web designer to use sign language in web page design. Since the system is web-based, if web designers are equipped with common computing environment for internet browsing, they can use the system. The web-based text-to-sign language system takes the format of bulletin board as user interface. When web designers write paragraphs and post them through the bulletin board to the translating server, the server translates the incoming text to sign language, animates with $3 \mathrm{D}$ avatar and records the animation in a MP4 file. The file addresses are fetched by the bulletin board and it enables web designers embed the translated sign language file into their web pages by using HTML5 or Javascript[6].

Kim, S. I. et al was focused on the inconvenience of location information, that the visually impaired experience the most, and the purpose of this study was to develop an innovative type of information that will provide the visually impaired with real time, indoor/outdoor location information. Due to the newly emerging RFID technology, online and offline environments are becoming innovative connected, and service usage costs or facility investment costs are being minimized so it can be applied on a national scale which created a new paradigm in the information service range as an advanced IT country. Moreover, it can also contribute to increasing the national compatibility as a type of knowledge based business[7].

Choi, J.H. et al., apply a context awareness based on Gaussian mixture model (GMM) to a sound reinforcement for hearing impaired. The harmful sound amplified through the sound reinforcement algorithm according to context awareness based on GMM which is constructed as Mel-frequency cepstral coefficients (MFCC) feature vector from sound data. According to the experimental results, the proposed approach is found to be effective in the various acoustic environments[8].

The purpose of these studies is to collect measured data from sensors and perceive context-information on daily life. The context-information using sensors can be used more actively, in the field of support for people with disabilities. Among people with disabilities, some have difficulties in communication. Many 
studies in various fields have been conducted to fully support routine communication of people with communicative disorders.

In addition, a research issue to be more immediately engaged is to enable communication of people with disabilities in urgent contexts. Urgent or hazardous situations may bring about lethal outcomes for them. Actually, the number of deaths is 0.8 for normal people, compared with 2.6 for people with disabilities, when a fire accident occurs, according to statistics in Korea, suggesting that the latter have difficulties in coping with urgent situations. Some actions against urgent situations include emergency communication for requesting rescue and transmission of urgent situations. Hence, it is necessary to study methods to quickly inform or perceive urgent situation, for a short time, rather than those to communicate complicated ones for enough time. Some studies on methods to quickly understand imminent crisis perceived by people with disabilities and transmit information about it. Therefore, the next chapter suggests methods to communicate in urgent situations, by using data from multi-sensors.

\section{Application of Internet of Things based on Sensor Data for Emergency Communication of Physically Disabled}

Disability is defined as a person who suffers from disability or difficulty in living in a daily life because of impaired disability or lack of mental abilities. When distinguishing between disabilities, the most significant are physical and mental disabilities. Disability disorders are divided into external disability failures and internal organs, disability disorders, disability impairments, deafness, deafness, deafness, and facial disorders.

Disability disorders refer to motor dysfunction and body dysfunction, and the disorder refers to a disorder in which the central nervous system muscles, bones, joints, and joints are unable to function themselves. Disability disorders include failure modes of paralysis, joint stiffness, amputation, and deformation.

Disability disorders are significantly restricted in other areas, except for impaired walking and daily life. Cerebral lesions refer to the physical and mental disorders caused by cerebral lesions such as cerebral palsy, strokes, traumatic brain injury, and traumatic brain injury.

There are always factors that threaten safety in everyday life. The modern society has been expanding its social capital and improved various policies and facilities to guarantee the welfare of citizen. There are three things that can pose a threat to life safety: natural disasters, social disasters, and crimes. Natural disasters are caused by natural phenomena such as floods, earthquakes, hail and heavy snow, and social disasters such as fires, gas explosions and traffic accidents are caused by abnormal operation of the human life, such as fires, gas explosions and traffic accidents. In the absence of such a hazard, a person who is free from daily life and a non-hardening disorder may suffer from difficulties in avoiding danger or harm to a person who is unable to avoid danger. In real life, the needs to communicate rapidly even though disabled people do not actually experience the risk situation.

If a person with a physical disability is aware of the danger and avoids the risk, or urgently needs to express his intention by urgent necessity, even if this communication can be difficult, a solution should be prepared to solve this problem.

In recent years, the combination of IT(Information Technology) technology and $\mathrm{CT}$ (Communication Technology) technology is called ICT technology and it is applied to solving problems in various fields. Various sensors are keys to the application of ICT technology. Sensors respond to and respond to physical changes, chemical changes, or biological changes depending on the function of the sensor during a change and when a phenomenon occurs. The sensor can convert this change to an electrical value, which can 
be expressed as a numerical value. An amplifier is indispensable because the difference in sensed change is detected only marginally.

When a person with a disability recognizes a sudden change of situation or wants emergency communication, he or she estimates the behavior or condition of a disabled person who wants to communicate and grasps what the disabled person wants to convey based on the estimation result. There is not a wide variety of expressions in case of an urgent situation or an urgent need to express a doctor. In Korea, elderly people living alone live alone to prevent loneliness. In this system, various sensors are used, and the communication method that the elderly person to be protected sends to the external help person is only pressing the emergency button. In order to protect the elderly people who want to protect their elderly people, it is necessary to monitor the elderly people by using GPS, But there are only very limited channels in which the elderly person is willing to externally display his or her intention by pressing and calling buttons.

\section{Communication goals}

In this study, it is an urgent way of expressing an opinion, and suggests the contents of the urgent communication and the emergency communication plan for disabled people. Emergency doctors can be classified according to their degree, and it is necessary for the communicating party to be able to recognize the emergency situation and to measure urgency.

\section{Communication methods and contents}

Communicating parties are persons with disabilities. The handicapped person here refers to a disabled person who is inconvenient or difficult to respond to a dangerous situation, and is significantly slower than his / her emergency degree in delivering emergency doctors or unable to communicate. It is important to identify the doctor you intend to communicate prior to communication. In this study, 3-axis acceleration sensor is used to confirm the intention to transmit. The 3-axis acceleration sensor is a sensor that detects the gravitational acceleration in the 3 -axis direction. The three-axis acceleration sensor is a sensor that has been standardized in the recently-popularized smart devices such as smart phones and smart watches. This study assumes that the disabled person, who is a communicator, has at least one smart device.

Smart devices can be used for communication network connection and information processing together with sensors. The procedure for confirming the doctor is as follows.

1) Obtain the value from the 3 -axis acceleration sensor.

2) Obtain the measured values of the 3-axis acceleration sensor continuously over time.

3) Separate time zones at regular intervals.

4) The measurement values of the 3 -axis acceleration sensor obtained at each time zone are classified by time zone.

5) Analyzes the change of measurement value in $x, y, z$ axis of each time zone.

6) Sum the measured values of each axis and sum the square root of the result.

7) Analyze the change of the processing result value 6) times in each time zone.

8) Converts the result of 6) to Fourier transform.

9) Analyze the results of 8) processing at each time zone.

10) Judge whether there is a statement about an emergency situation

11) Emergency communication When there is an intention to communicate, the emergency degree is judged.

12) Send an emergency doctor.

The value $x_{n t}, y_{n t}, z_{n t}$ comes in from the sensor as $X_{n t}=\left\{x_{n 1}, x_{n 2}, \cdots, x_{n t-1}, x_{n t}\right\}$.

$$
D S_{(n t, T)}=\left\{X_{n t}, Y_{n t}, Z_{n t}, T\right\}
$$

$D S_{(n t, T)}$ is the $n$th dataset of length $T$ received at time $t$. 
$X_{n t}, Y_{n t}, Z_{n t}$ is a set of $\mathrm{x}, \mathrm{y}, \mathrm{z}$ axis acceleration sensor data values at the time $\mathrm{t}$.

And updates the $D S_{(n t, T)}$ function when the predetermined interval $\mathrm{T}$ is exceeded. $X_{n t}, Y_{n t}, Z_{n t}$ are updated according to the update $D S_{(n t, T)}$. Norm is as follows.

$$
N=\sqrt{x_{n t}^{2}+y_{n t}^{2}+z_{n t}^{2}}
$$

Now the Fourier transform of Norm $F(f)=\int N(t) e^{-2 \pi i f t} d t$ is added and the following is calculated from the obtained result.

Let $F_{n}$ be the solution of $\frac{d F(f)}{d f}=0$ from this function. This $F_{n}$ value means the value of the peak appearing in the Fourier transformed region.

$$
V_{n}=\sum_{f \subseteq\left(F_{n}-\delta, F_{n}+\delta\right)}\left(F_{n}-F(f)\right)^{2}
$$

The above function computes the variance value for the region within the range $2 \delta$ near the peak.

If this variance exceeds the limit we have set arbitrarily, that is, if there is a clear indication that the user is in an irregular motion, the emergency level is increased by the irregular level. Suppose that the irregular level takes a standard deviation.

if $V_{n}>\alpha_{t h}, \gamma_{t}=\gamma_{t-1}+\sqrt{V_{n} /(2 \delta)}$

else, $\gamma_{t}=\gamma_{t-1}-1$

Therefore, after analyzing the Fourier result of the corresponding time zone, the newly emerged motion condition is recognized and the total urgency level is determined.

The pseudo code of the suggested algorithm is written below.

\section{Table 1. The Pseudo Code of Algorithm}

Input: 3-axis acceleration sensor data, time output: judgement for an emergency situation

\#Define functions get_accel_data(sensor):

collect data from the 3 -axis acceleration sensors

return (data_x, data_y, data_z, time)

separate_data(sensor_data):

if there is no timeslot for sensor_data from this second: make new timeslot

else:

classify sensor data for each timeslot

return timeslot \#this timeslot contains sensor data for few seconds

Analysis_change(data list):

for all data in datalist: calculate differential(data)

\#find pattern

find period of data

find period of $\left(\frac{d}{d x}\right.$ data $)$

find the point of $\frac{d}{d x}$ data $=0$ 
return pattern(data list)

Geometric_sum(timeslot):

for all data in timeslot:

data_avrg $=\operatorname{sqrt}($ data_x $. \wedge 2+$ data_y.^2 + data_z.^2)

return list of data_avrg

$\mathrm{i}=0$

\#Body of the algorithm

While True:

Timeslot $[\mathrm{i}]=$ separate_data(get_accel_data(sensor) $)$

Analysis_change([list of data_x in Timeslot[i]])

Analysis_change([list of data_y in Timeslot[i]])

Analysis_change([list of data_z in Timeslot[i]])

Geometric_sum(Timeslot[i])

Analysis_change([list of data_avrg in Timeslot[i]])

FFT_list = Fast_fourier_transform([list of data_avrg in timeslot $]$ )

Analysis_change(FFT_list)

if pattern $($ Timeslot $[i]) \neq$ pattern(Timeslot $[i-1])$ :

There is an emergency situation

$\mathrm{i}=\mathrm{i}+1$

end of while

\section{Advantages of the proposed method}

The emergency communication method proposed in this study does not require additional terminals. The discomfort or discomfort incompatibility of using an additional handset is greater than that of a non-handicapped person. The use of sensors embedded in existing smart devices is advantageous in that the inconvenience and heterogeneity of users with disabilities can be minimized.

Next, it can be applied to a wide range of people with disabilities. It can also be applied to persons with speech impairments that are not normally communicable, or persons with difficulties in manipulating the interface of communication equipment. This is because the proposed method is based on the behavior of a user with a disability who wishes to communicate and analyzes the motion pattern according to the passage of time.

\section{Experiment and Evaluation}

In this chapter, we summarize and analyze and evaluate the results of experiments on the items proposed in Chapter 3. Experiments performed in this study use a 3 - axis acceleration sensor built into the Smartphone. The values received from the three-axis acceleration sensor were stored as three kinds of values according to the passage of time and analyzed again. The following figure is a graph showing measured values from 3 -axis acceleration sensor according to time. This graph shows how the behavior changes as you change certain actions. The change in a particular axis is large and the change in the other axis is insignificant. 


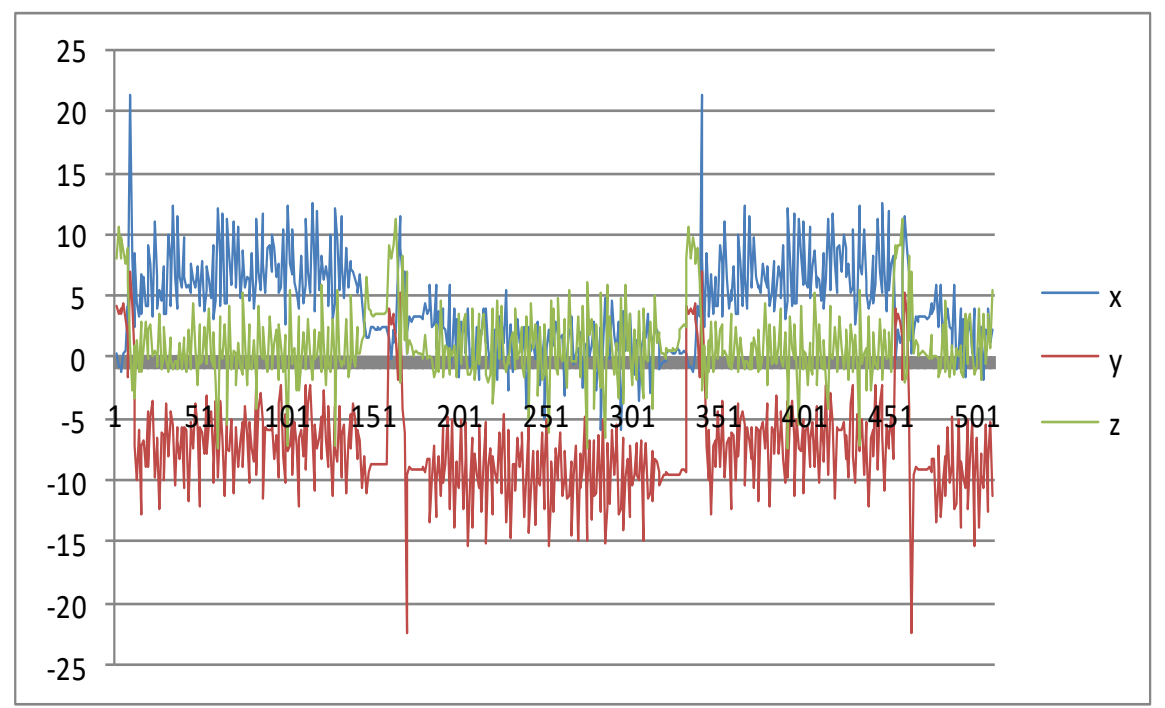

Figure 1. 3-axis Acceleration Sensor Value

The next graph is to square the values of the respective axis, add the squares, and then find the square root again. Directional components were removed and values representing the magnitude of the momentum were calculated. This graph also shows a different pattern depending on the behavior. However, the change of the activity amount can be confirmed. The size of the activity can be inferred through this graph, which can infer the present state of communicating the handicapped person and get a clue to deduce urgent doctors who want to communicate.

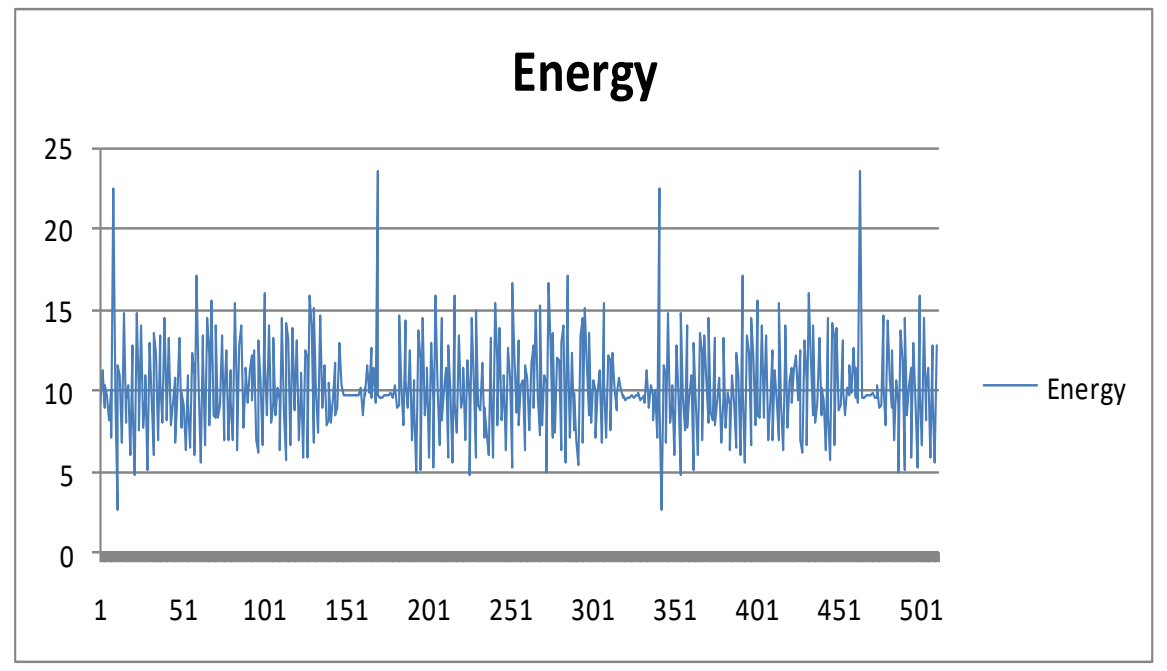

Figure 2. Energy Conversion of 3-axis Acceleration Sensor Value

This graph is a graph of the values obtained by Fourier transforming the above values. It can be seen that no change occurs in a specific operation. Instead, changes are taking place in meaningful changes. In other words, it can be seen that an event occurs when the robot starts to move in a stopped state and when it is changed from a regular motion state to an irregular motion state. 


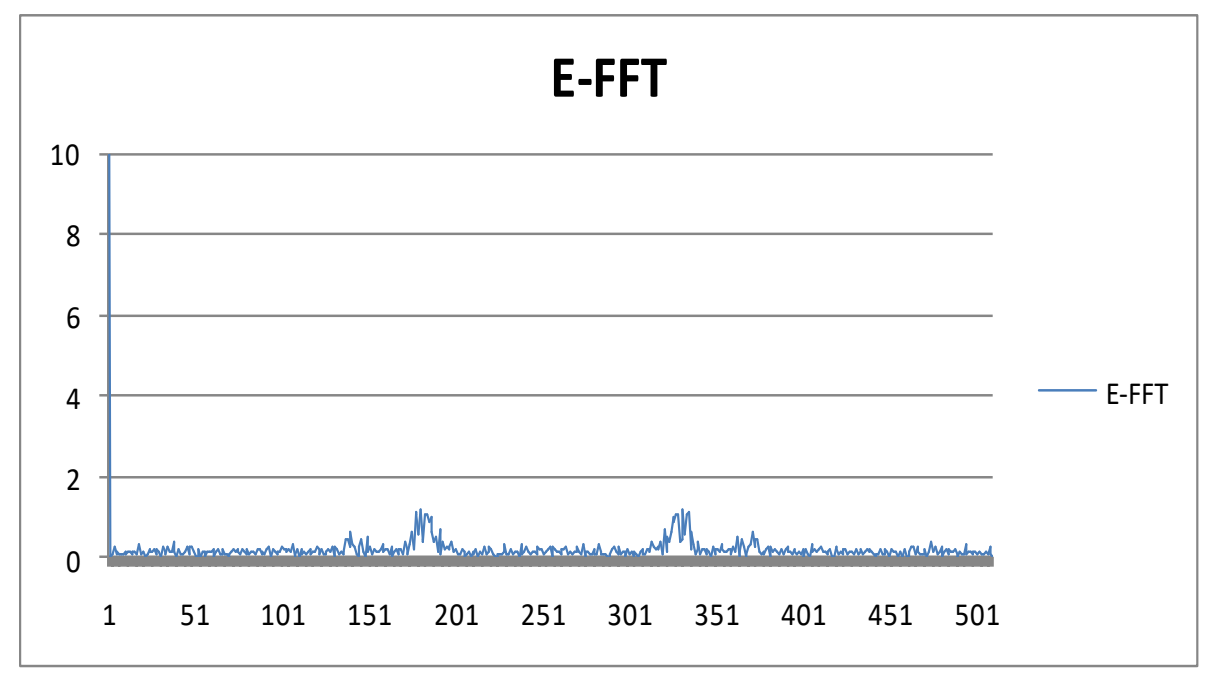

Figure 3. Converting Fourier Transform Values of Energy Values

Using these characteristics, it is possible to summarize the intention to deliver urgently as follows.

Conversion value event occurrence No-Regular Regardless of exercise status, normal.

Conversion value event occurrence - Regular motion: Normal conversion value event occurrence - Irregular motion: Abnormal.

Conversion value event occurrence - Irregular motion state repetition: Emergency rescue request.

\section{Conclusion}

Some people with disabilities have difficulty communicating in an emergency situation, so they are in danger of being seriously injured by failure to communicate urgently, such as an emergency rescue request. In this study, we propose a method to utilize 3 - axis acceleration sensor based on the Internet of things to make urgent communication possible. The measured values of the three-axis acceleration sensor built in the generalized smart device such as smart phone or smart watch are obtained according to the time and classified by time. By making three measurement values of the three-axis acceleration sensor and comparing them by time zone, the disabled people inferred the urgent communication intention by recognizing the behavior pattern in the urgent situation and the intelligent propagation. Future research will need to be able to embody ideas in this research and apply them to actual applications.

\section{Acknowledgments}

This work was supported by the Ministry of Education of the Republic of Korea and the National Research Foundation of Korea(NRF-2016S1A3A2925520)

This paper is a revised and extended version of a paper entitled [Sensor Data Utilization for Urgent Communication of Physically Handicapped Persons Based on the Internet of Things] presented at [FGIT2017, Daejeon University, Korea, December21-23, 2017]. 


\section{References}

[1] C.Y. Lim, K.M. Jeon, K.H. Kim, K.C. Ko and K.N. Koh, "A Study on the Estimation of Energy Expenditure and falls measurement system for the elderly", J. The Korea Society of Computer and Information, vol.17, no.4, (2012), pp.1-9.

[2] K.M. Jeon, K.C. Ko, H.M. Lee and Y.H. Kim, "Development of Textile Fabrics Flexible Platform based Multiple Bio-Signal Central Monitoring System for Emergency Situational Awareness in High-Risk Working Environments", J. The Korea Society of Computer and Information, vol.19, no.12, (2014), pp.227-237.

[3] J.S. Kim, J.Y. Kim and W.C. Lee, "Implementation of Indoor Positioning System for Visually Impaired People Using Android Smartphone and Visible Light Communication", JKIIT, vol.13, no.1, (2015), pp.87-92.

[4] K.W. Kang and T.S. Kimn, "Development of Human-machine Interface based on EMG and EOG", J. The Institute of Electronics and Information Engineers, vol.50, no. 12, (2013), pp.129-137.

[5] C.S. Kang, H.S. Jo and B.H. Kim, "A Machine-to-machine based Intelligent Walking Assistance System for Visually Impaired Person", J. of Korea Information and Communications Society. vol.36, no.3, (2011), pp.287-296.

[6] S.W. Park and B.H. Wang, "Web-based Text-To-Sign Language Translating System", J. of The Korean Institute of Intelligent System, vol.24, no.3, (2014), pp.265-269.

[7] S.I. Kim, "System Development for Location Information for the Visually Impaired: Mainly Regarding the Development of an Integrated On Off Line", Archives of Design Research, vol.20, no.5, (2007), pp.217-228.

[8] J.H. Choi and J.H. Chang, "Sound Reinforcement Based on Context Awareness for Hearing Impaired", J. of the Korean Institute of Telematics and Electronics. C, vol.48 SP, no. 5, (2011), pp.109-114.

[9] S.J. Lee, Y.R. Shin and E.N. Huh, "A study on the measurement method for the walk of patients with Parkinson's disease using 3-axis acceleration sensor", J. Korea Institute of Communication and Information Science (Summer 2012), (2012), pp.820-821.

[10] G.S. Ha, H.S. Lho and W.D. Cho, "Step Count Detection Algorithm Using a 3-Axial Accelerometer Sensor", J. Korea Institute of Communication and Information Science (Fall 2012), (2012), pp.151-152.

\section{Authors}

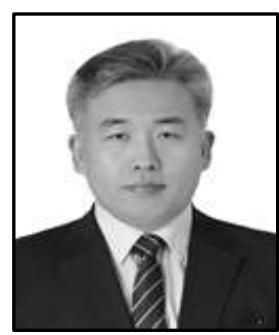

Donghyeok Suh, he is a professor at Dankook University. He received the M.S. degrees in computer engineering from Hoseo University in 2005 and the Ph.D. in computer science from Chungbuk National University in 2012. His research interests included in stream data processing and data fusion in wireless sensor network.

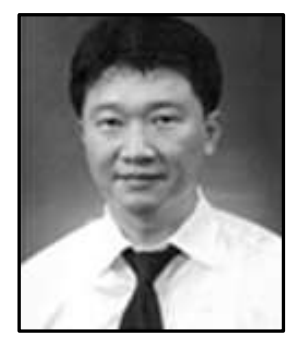

Chang-Won Seo, he is a professor at Far East University. He received the B.A. degree in special education from Taegu university in1994 and the M.A. degree in 2002. He received the Ph.D. from Seoul National University. His research interests included communication tool.

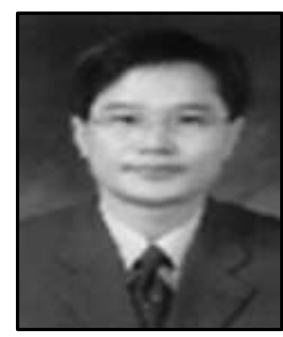

Kwang-Chul Park, he obtained a BA in English Literature and Linguistics from Inha University, Korea in 1991. He also obtained a $\mathrm{Ph}$. D in Linguistics form Indiana University at Bloomington in 2003. $\mathrm{He}$ is a professor in the Department of Global Secretarial Administration at Korea Nazarene University. He is interested in second language acquisition and sign language development. 
International Journal of Control and Automation

Vol. 11, No. 3 (2018) 